\title{
Construction Strategy of Regional Plant Landscape in Urban Gardens
}

\author{
Jinwen Chen ${ }^{1}$, Weiran Tian², Ying Huang ${ }^{2, *}$ \\ ${ }^{1}$ Guilin Landscape Planning and Architecture Design Research Institute, Guilin 541002, China \\ ${ }^{2}$ College of Tourism\&Landscape Architecture, Guilin University of Technology, Guilin 541004, China
}

\begin{abstract}
By sorting out the problems existing in the construction of plant landscapes in urban gardens, the designer plans tree species, characteristics, spaces, colours, etc. from the perspective of ecosystem balance, applies local rich native plants, and explores the construction strategies of regional plant landscapes in urban gardens. Taking the city of Guilin as an example, the article analyses the construction features of the band green landscape of the two rivers and four lakes scenic spot, summarizes the construction characteristics of the regional plant landscape of Guilin, promotes the construction of an ecological garden city, and meets the people's beautiful environmental needs.
\end{abstract}

\section{Introduction}

The plant landscape is an important bridge connecting other ecological factors in the natural world, and plays an important role in protecting the existing biological chain and repairing the damaged urban landscape. The designer uses the morphological characteristics, colours and texture of plant materials to form a threedimensional space with a certain length, width and thickness and uses the dynamic sequence changes of plant time and space to form a plant landscape with distinctive characteristics, rich seasonal changes, and strong integrity, thus highlighting the characteristics and creativity of urban gardens. This article considers the urban ecosystem and combines the natural landscape and artificial landscape in the urban area to form a balanced natural ecosystem. Taking the scenic spots of two rivers and four lakes in Guilin as an example, the plant landscape composition of different site conditions is analysed to create an all-around urban plant landscape in harmony with the landscape pattern of Guilin, in order to provide reference for the plant landscape construction of urban gardens.

\section{Carding problems of plant landscape construction in urban gardens}

\section{1 lgnoring the protection of natural plant landscapes}

Large squares and large lawns seem to have become symbols of some urban styles. Urban construction is destroying the original vegetation and creating monotonous plant landscapes, focusing only on the beauty of the landscape and ignoring the ecological benefits, which have an adverse impact on the urban environment and people's health. The ecological environment system is holistic, so designers ensure the continuity of natural vegetation to the greatest extent and build plant landscapes that meet the requirements of the ecological environment to achieve the harmonious integration of natural landscapes and urban landscapes. Generally, the mountains and hills in the city are covered with dense vegetation; rivers, lakes, and wetlands grow aquatic plants. The designer should fully protect the native natural vegetation, and retain the integrity and uniqueness of the native plant landscape to maintain the traditional local landscape and the balance of the ecological environment.

\subsection{Insufficient local and regional manifestation of plant landscape}

In recent years, new plant materials and new design concepts have been widely used in the urban landscape update, which have greatly improved the appearance of the city. At the same time, there have also been some tendencies to converge. The problem of "one side of the city" has become increasingly prominent. Assimilation and non-characterization are eroding every corner of the city. Villages are losing their regional, cultural and diverse landscape characteristics. Native plants are landscaping materials with strong growth, strong resistance and low cost in this area. They are rich in resources and diverse in form, and can constitute the characteristic plant landscapes in different regions. Applying these most "forgotten" and most common plants to the construction of urban garden landscapes has both a certain rural interest and an ecological feeling.

\footnotetext{
* Corresponding author: 490498081@qqq.com
} 


\subsection{The lack of humanized space in the plant landscape}

People choose a beautiful city just for a better life. If the urban garden plant landscape is limited to practical functions, but lacks humanistic care, humanized space is insufficient. Ignoring the quiet space, private space, communication space, etc. created by plants is not in line with the environmental construction of beautiful cities.

\section{Regional plant landscape planning}

\subsection{Tree species planning}

Plant material is the foundation of plant landscape construction. The selection of tree species is dominated by local native tree species, highlighting the geographical features, emphasizing the rational use of plant resources and biodiversity protection, and combining fast-growing and slow-growing tree species, evergreen trees and deciduous trees. The designer should choose a tree species that can fully express the local characteristics, reflect the urban style, and can serve as an important symbol of the city as the key tree species, such as Cinnamomum camphora, Ficus microcarpa, Osmanthus fragrans and so on. The designer should also select the tree species with excellent characteristics, high frequency of occurrence in various green spaces, large number of use, and development potential as the backbone tree species $^{[1]}$, such as Ginkgo biloba, Bischofia javanica, Ficus virens, Liquidambar formosana, Livistona chinensis and so on. In addition, according to the principle of biodiversity, good tree species can be used as general tree species, and under the premise of making full use of local tree species resources, some edge tree species can be introduced according to different green space types to increase the colour of the landscape, further enrich the urban plant landscape and improve ecological and environmental benefits ${ }^{[2]}$.

\subsection{Featured planning}

As an important content of plant landscape construction, characteristic planning is the key to reflect the characteristics of the site. According to the function and theme positioning of the site, the unique landscape space is created by using the shape, ornamental value and cultural characteristics of plants. For example, the city of Guilin with karst landforms is characterized by landscape gardens, forming an urban landscape pattern that blends mountains and waters and blends city views. Relying on the unique natural landscape, the plant landscape is based on the protection of natural karst plant resources, simulating natural vegetation communities, using the plant landscape to improve the natural transition between rivers, lake ecosystems, karst landscapes and the urban human environment, and maintaining the ecosystem stability and balance.

\subsection{Spatial planning}

The plant space is the smallest constituent unit in the garden green space, using the combination of garden plants to create different types of space such as open space, semi-open space, vertical space and covered space. Through the comparison and change of plant space, the designer builds beautiful plant landscapes with multiple layers of sense ${ }^{[3]}$, providing people with places to watch, tour, relax and entertain. In urban gardens, the humanoriented design of human-oriented plant space helps improve the quality of the urban environment and helps people's mental and physical health. According to human behaviour and psychological characteristics, the designer provides high-quality plant space to meet the practicality and suitability requirements of people for space.

\subsection{Colour planning}

Colour is the most direct and sensitive content for plant landscape appreciation ${ }^{[4]}$. People have different psychological feelings about plant landscape colour. In plant landscape planning, plant landscaping is based on colour characteristics to create a more humane and scientific plant landscape. Plant landscape colour planning determines the tone and layout of plant colours from a macro level, and clarifies the theme of plant landscapes ${ }^{[5]}$. In addition, the timing caused by the seasonal changes of plants is irreplaceable by other components. For example, Guilin is in the midsubtropical zone, and its vegetation is evergreen broadleaved forest. The plant landscape in urban gardens is dominated by green, with red, yellow, purple, etc. as the colour matching, highlighting the colourful four-season plant landscape. The designer plants flowering plants in the red series in the spring, such as the red flowering Bauhinia variegata, Amygdalus persica'Duplex', Cerasus serrulata, etc. The designer matches the red and purple flowering plants in the rich green shade of summer, such as Lagerstroemia indica, Nerium oleander, Punica granatum, etc. The designer plants colourful deciduous plants in the yellow and red series in autumn, such as L.formosana, G.biloba, Metasequoia glyptostroboides, Triadica sebifera, Koelreuteria paniculata and so on. In winter, evergreen plants are still lush, dotted with red, yellow and other flowering plants, such as Camellia petelotii, Armeniaca mume, Chimonanthus praecox and so on. Some colour-leaf plants such as Duranta erecta'Golden Leaves', Prunus cerasifera f. atropurpurea, and Excoecaria cochinchinensis also add colour to the scenic spot.

\section{Case study - - strategies for plant landscape construction in two rivers and four lakes scenic spot in Guilin}

\subsection{Base overview}

Guilin two rivers and four lakes scenic area is located in the central area of Guilin. It consists of Rong Lake, Shan Lake, Gui Lake, Mulong Lake, and the Lijiang River and Taohua River flowing through the urban area. The water 
area of the whole area is $38.59 \mathrm{hm}^{2}$, the ecological revetment is $10.60 \mathrm{~km}$ long and $10-30 \mathrm{~m}$ wide, and the green land on the lakeshore is distributed in a strip. Guilin two rivers and four lakes scenic area is the largest urban open park in Guilin. It is an important place for citizens and tourists to relax, watch, relax and entertain. It is also the "green lung" and tourist cultural symbol of Guilin. The green space of the scenic spot is distributed in a band shape, close to the mountain and the water, and adjacent to the urban landscape.

\subsection{Strategies for regional plant landscape construction}

The plant landscape planning of the Guilin two rivers and four lakes scenic spot combined with the site topography and overall layout constitutes a "four areas, two lines and multiple points" landscape structure. "Four areas" refer to the four major plant areas formed around Shan Lake, Rong Lake, Gui Lake, and Mulong Lake

(Figure 1); the first line is the coastal waterfront plant landscape zone, and the other line is adjacent to the city green landscape line. The various landscape nodes connected in series.

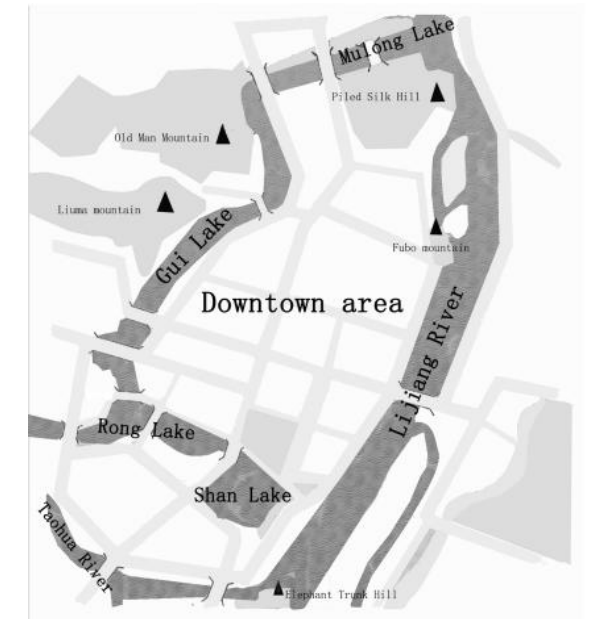

Fig. 1. Schematic diagram of Guilin two rivers and four lakes scenic spot.

\subsubsection{Landscape features of planar green space}

The Guilin two rivers and four lakes scenic area takes water as the center of the landscape and has excellent natural conditions. Various plants are planted along the shore of the lake. Therefore, the landscape plant landscape structure of the scenic spot is divided into four sub-areas of Shan Lake, Rong Lake, Gui Lake and Mulong Lake according to the geographical conditions of the site, and each sub-area has distinctive plant landscape features (Table 1).

(1) Shan Lake district. The plant landscape of Shan Lake is centered on the theme of "cedar". On the basis of the original small amount of Metasequoia, the designer planted Taxodium distichum, T.distichum var. imbricatum, and M. glyptostroboides in the water and wet environment, either planted on the shore, or with other plants. Trunk straight and soft texture of the metasequoia forest and the open horizontal lake form a strong contrast in the direction, forming a unique lakeshore landscape.

(2) Rong Lake district. The plant landscape of Rong Lake takes the theme of "Ficus". The millennium ancient banyan planted in front of the ancient south gate has a tree height of $19 \mathrm{~m}$, a breast diameter of $1.62 \mathrm{~m}$, and a canopy coverage of $1056 \mathrm{~m}^{2}$. The tree is beautiful in shape, the branches and leaves are connected, the roots are pulled together, and the single tree is a forest, which is spectacular. The green area next to the ancient banyan is arranged with groups of banyan trees. Banyan trees of different shapes echo the orphaned ancient banyan. The crowns of the trees are joined together to form an independent ancient banyan square. The ancient banyan and the mottled ancient city wall tell the long history of Guilin.

(3) Gui Lake district. The plant landscape of Gui Lake runs through the whole garden with the osmanthus which is the "city flower" of Guilin. The coastal landscape is composed of native plants such as G. biloba, F. microcarpa, Y. denudata, Keteleeria davidiana var. calcarea, and Trachycarpus fortunei. It has many types of plants and various combinations of shrubs. Threedimensional plant configuration and seasonal changes reflect the characteristics of biodiversity. The Expo Park not only has an ornamental role, but also shows important science value.

(4) Mulong Lake district. With the theme of Song culture, Mulong Lake builds ancient buildings such as the ancient Song city, Mulong tower, and Tinghexuan, reflecting the "past, present, future" urban cultural connotation characteristics. In plant landscaping, the city pays attention to the coordination of plants and buildings. Outside the city, taking Guilin mountains and rivers as the background, on the basis of retaining the original vegetation, it simulates the local natural ecological community to form an ecological plant landscape that integrates natural and artificial, environmental and historical culture.

Table 1. Main characteristic of plant landscape in Guilin two rivers and four lakes scenic spot.

\begin{tabular}{|c|c|}
\hline Partition & Featured plant landscape \\
\hline Shan Lake district & $\begin{array}{c}\text { M. glyptostroboides + T. } \\
\text { distichum + T. distichum - } \\
\text { Ophiopogon japonicus }\end{array}$ \\
\hline Rong Lake district & $\begin{array}{c}\text { F. microcarpa - Philodendron } \\
\text { selloum + Colocasia esculenta } \\
\text { - O. japonicus }\end{array}$ \\
\hline Gui Lake district & K. davidiana + Cedrus \\
& deodara - Acer palmatum \\
& 'Atropurpureum' - O. \\
japonicus
\end{tabular}

\subsubsection{Landscape features of banded green space}


Guilin two rivers and four lakes scenic area is a beltshaped waterfront park with a short axis and no main roads. The winding roads connect various landscape nodes in series to form a variety of waterfront plant landscapes. The planting of garden roads is planned according to the theme of each functional division.

The plant space forms on the waterside are mainly open and semi-open spaces, which adopt water-resistant and wet plants such as Metasequoia, palm elm and weeping willow to form an orderly opening and closing and varied waterfront landscape. Gui Lake scenic area has the longest coastline, and uses plants with local characteristics of Guilin to build characteristic plant landscape gardens, such as palm gardens, magnolia gardens, banyan tree gardens, ginkgo gardens, coniferous tree gardens, etc., which fully demonstrate the regional landscape characteristics.

The plant landscape near the mountain should be coordinated with the karst topography. The plant landscape mainly simulates the construction of natural plant communities. While protecting the natural vegetation on the stone mountain, the artificially created garden plant community at the foot of the mountain is closely integrated with the natural plant community to form a harmonious and beautiful landscape. The designer selects tree species from the perspective of the actual situation of the site and the ecological balance to create a relatively stable and diverse plant community.

The plant space on the side adjacent to the urban landscape is mainly closed. The plants form a natural green barrier to isolate the hustle and bustle of the city, directing visitors' sights to the water and admiring the beautiful lake and mountain scenery. For example, designer uses forest planting Metasequoia to create the "green wall" of the city, the sidewalk camphor tree is also a good green barrier. These trunks are straight and complete, and the famous ornamental tree with variable leaf colour is called the beautiful landscape of the city.

In the construction of plant landscapes in Guilin city, starting from the overall layout, combined with the topography of the site, according to the local conditions, the distant green hills and nearby green water are organically unified with the urban landscape to form a landscape pattern of mountains and rivers.

\section{Conclusion}

The designer uses native plants to create regional urban garden landscapes, highlights urban characteristics, and enhances urban environmental quality. The urban garden plant landscape forms a landscape effect that changes in unity and seeks unity in change. Let the native plants be better presented in our lives, making the urban landscape more beautiful, and making our lives better.

\section{Acknowledgement}

We thank the National Key R\&D Program of China (2019YFC0507503) for funding support.

\section{References}

1. D.K. Zang. Landscape Architecture [M]. Beijing: China Architecture \& Building Press. 2012.

2. L.J. Wang. Investigation and Distribution, Planning on the Garden Trees in Urmqi City [J] . Chinese Agricultural Science Bulletin,2010,26 (18) :128132.

3. Y.Y. Zhang, L.H. Xu, Y.J. Shi, et al. Research Progress on Space Construction Methods of Landscape Plants [J].Northern Horticulture, 2020(09):150-156.

4. C.J. Li, P.Y. Jia, L. Dong. Research on the Procedures and Methods of Planting Plan and Design [J].Chinese Landscape Architecture, 2014,30(01):93-99.

5. T.Yan. On the Planning and Design Method of the Modern Plant Landscape [J]. Chinese \& Overseas Architecture, 2014(10):69-71. 